\title{
EFFECT OF LEPTIN CONCENTRATIONS AND LEPTIN RECEPTOR GENE POLYMORPHISMS ON THE OUTCOME OF RENAL TRANSPLANTATION
}

\section{Type}

Research paper

\section{Keywords}

leptin, leptin receptor, Renal transplant, genetic polymorphisms

\begin{abstract}
Introduction

Leptin is a pro-inflammatory adipocytokine implicated in cardiovascular disease, insulin resistance, obesity and chronic kidney disease.

Material and methods

In a cohort of 236 renal transplant recipients, we aimed to determine whether circulating leptin concentrations and/or three polymorphisms in the leptin receptor (LEPR) gene, namely rs 1137100 , rs 1137101 and rs1805094, were related to clinical outcomes in renal transplantation. Plasma leptin concentrations were measured by ELISA. Genetic variants were determined by conventional realtime PCR assays and statistical associations with clinical outcomes were obtained by logistic regression modelling.
\end{abstract}

\section{Results}

Patients with elevated leptin levels were at higher risk of acute rejection [OR=1.03 (1.01-1.05), $p=0.03$ ] and displayed worse renal clearance $(p=0.001)$ than patients with lower levels. Leptin levels were not significantly affected by any of the three LEPR SNPs. The rs $1137101 \mathrm{G}$ variant showed an inverse association with the risk of delayed graft function (DGF) [OR=0.42 (0.22-0.81), $p=0.009$ ], whilst the homozygous rs1805094 CC genotype was associated with increased risk of acute rejection [OR=11.38 (2.15-60.16), $p=0.004]$. A statistically significant association was also observed between the rs $1137100 \mathrm{GG}$ genotype and better renal function [mean difference vs. AA/AG $=20.20$ (4.91-35.49) $\mathrm{ml} / \mathrm{min}, \mathrm{p}=0.010]$.

\section{Conclusions}

Our results show that both leptin plasma concentrations and polymorphisms in the LEPR gene may affect clinical outcomes in renal transplant recipients, suggesting that the determination of these parameters could be useful in predicting post-transplant adverse events. 
EFFECT OF LEPTIN CONCENTRATIONS AND LEPTIN RECEPTOR GENE POLYMORPHISMS ON THE OUTCOME OF RENAL TRANSPLANTATION

Running head: Leptin levels and SNPs in renal transplant 


\section{ABSTRACT}

Introduction: Leptin is a pro-inflammatory adipocytokine implicated in cardiovascular disease, insulin resistance, obesity and chronic kidney disease.

Material and Methods: In a cohort of 236 renal transplant recipients, we aimed to determine whether circulating leptin concentrations and/or three polymorphisms in the leptin receptor (LEPR) gene, namely rs1137100, rs1137101 and rs1805094, were related to clinical outcomes in renal transplantation. Plasma leptin concentrations were measured by ELISA. Genetic variants were determined by conventional real-time PCR assays and statistical associations with clinical outcomes were obtained by logistic regression modelling.

Results: Patients with elevated leptin levels were at higher risk of acute rejection [OR=1.03 (1.01-1.05), $p=0.03]$ and displayed worse renal clearance $(p=0.001)$ than patients with lower levels. Leptin levels were not significantly affected by any of the three LEPR SNPs. The rs1137101 G variant showed an inverse association with the risk of delayed graft function (DGF) [OR=0.42 (0.22-0.81), p=0.009], whilst the homozygous rs1805094 CC genotype was associated with increased risk of acute rejection [OR=11.38 (2.15-60.16), $p=0.004]$. A statistically significant association was also observed between the rs1137100 GG genotype and better renal function [mean difference vs. $A A / A G=20.20(4.91-35.49) \mathrm{ml} / \mathrm{min}, \mathrm{p}=0.010$ ]. Conclusions: Our results show that both leptin plasma concentrations and polymorphisms in the LEPR gene may affect clinical outcomes in renal transplant recipients, suggesting that the determination of these parameters could be useful in predicting post-transplant adverse events.

Keywords: leptin, leptin receptor, renal transplant, genetic polymorphisms 


\section{INTRODUCTION}

Kidney transplantation is considered as the best treatment option for patients with end-stage renal disease and the knowledge of the immune system is crucial in its management [1]. Among the organs related to the immune system, the adipose tissue is gradually gaining importance. Thus, in the last few years, this tissue, which was believed to simply function as energy storage, has also been acknowledged an important role in endocrine and immune homeostasis by synthesizing a variety of hormones, known as adipocytokines [2]. Leptin is one of the most widely studied of these adipocytokines. It has been implicated in the pathogenesis of cardiovascular disease (CVD), through a crosstalk between adipose tissue and blood vessels, as well as being associated with inflammation and NO production [3]. Patients with chronic kidney disease (CKD) have been shown to have increased circulating levels of leptin that may result from an increase in their systemic production and/or a decrease in their renal excretion [4-7]. In these patients, higher leptin levels have been related to coronary heart disease [8], hypertension [9] and diabetes mellitus [10]. Much less is known, however, about the role of leptin in renal transplant recipients. The few reported results in these patients reveal findings that do not necessarily reflect the same situation as in the general population $[11,2,12]$. In the same manner, how and why leptin levels fluctuate after grafting is not completely understood yet.

The leptin receptor, coded by the LEPR gene, is present in the cell membranes of a wide variety of cells of different tissues, indicating the critical physiological functions of this adipocytokine [13]. In this regard, a number of single nucleotide polymorphisms (SNPs) in this gene have been suggested to play an important role in different morbid conditions such as diabetes, hypertension and other CV events $[14,15,12,16]$, all of which are commonplace in renal transplant recipients. 
In the present study we have aimed to determine (i) whether three common, functional SNPs in the LEPR gene (Lys109Arg rs1137100, Gln223Arg rs1137101 and Lys656Asn rs1805094) were related to different outcomes in renal transplantation, (ii) whether leptin concentrations were associated with these outcomes and (iii) whether these SNPs may affect such concentrations in a population of renal transplant recipients.

\section{PATIENTS AND METHODS}

\subsection{Study design}

The study sample consisted of 236 Caucasian patients who underwent kidney transplantation at the Badajoz University Hospital, the reference center for renal transplantation in the health district of Badajoz (Southwest Spain). Patients included in the study received a single kidney from deceased donors. We recruited all prevalent kidney transplant recipients who attended the Transplant Follow-up Unit between October 2014 and September 2015 and whose time since grafting was between one and fifteen years. At the time of their visit, patients received detailed information regarding the study and written informed consents were obtained. Fifteen-ml blood samples were then collected from all participants into BD Vacutainer EDTA tubes (Becton Dickinson, New Jersey, USA); $10 \mathrm{ml}$ were destined to DNA purification and the other $5 \mathrm{ml}$ were immediately centrifuged to obtain plasma for the determination of leptin levels. Both plasma and whole blood samples were then stored at -80 ㅇ $\mathrm{C}$ until analyzed. The study was approved by the Ethics Committee of the Badajoz University Hospital and the Bioethics Committee of the University of Extremadura and was conducted in accordance with the Declaration of Helsinki and its subsequent revisions. 


\subsection{Variables and clinical outcomes}

Medical records were retrospectively reviewed to retrieve meaningful clinical and demographic variables and different outcomes to assess their association with genetic polymorphisms and leptin levels. Delayed graft function (DGF) was defined as the need for dialysis within the first week after transplantation [17]. Acute allograft rejection was established by histological findings in renal biopsies according to the Banff classification and/or by clinical evaluation as previously described $[18,19]$. Renal function was assessed one year after grafting and at the time of sampling by using the Modification of Diet in Renal Disease (MDRD) formula [20] as suggested by the K/DOQI Group [21].

\subsection{Genotype analysis}

We used a standard phenol/chloroform extraction method in order to isolate genomic DNA from the whole blood samples. Three variants in the leptin receptor (LEPR) gene, namely rs1137100, rs1137101 and rs1805094 were identified by real-time PCR using commercial TaqMan ${ }^{\circledR}$ SNP Genotype Assays from Thermofisher Scientific (Rockford, II, USA). These SNPs were selected based on existing reports linking their presence to significant clinical repercussions. Previously sequenced samples were used as negative and positive controls to rule out possible genotyping errors. Likewise, the analysis of $5 \%$ of the samples was duplicated and confirmed by direct sequencing (ABI3700 DNA Analyzer; Perkin-Elmer/Applied Biosystems).

\subsection{Determination of leptin concentrations}

Plasma leptin concentrations were measured using immunoassay kits based on solid-phase sandwich enzyme-linked immunosorbent assay following the manufacturer's instructions 
(DRG Leptin Sandwich ELISA, DRG Instruments GmbH, Germany). A four-parameter curve fit was used for the calculation of the concentrations.

\subsection{Statistical analyses}

Chi-square or Fisher's exact tests were used for the univariate analyses of differences between categorical variables. Differences between quantitative variables across groups were assessed by Mann-Whitney/Kruskal-Wallis or T-student/ANOVA tests, depending on the normality of the data and the number of groups studied. The analysis of the effect of both genetic and nongenetic variables was performed with multivariate regression modelling, adjusting for demographic and clinical covariates. These variables were chosen because of their significance observed in univariate studies $(p<0.15)$ and/or based on clinical criteria. The covariates considered in each case are listed either in the Results section or in the related tables. For the haplotype study, the SNPassoc package in the $R$ environment was utilized to estimate the frequency of the resulting haplotypes and to determine the effect of these allele combinations on the analyzed outcomes controlling for significant covariates. Frequency threshold for rare haplotypes was set at 0.01 .

Statistical analyses were performed using the SNPassoc $R$ package and the IBM SPSS Statistics package v.22 (IBM Corporation, Armonk, NY, USA).

\section{RESULTS}

The study comprised 236 renal transplant recipients, 143 (60.6\%) of whom were males; their mean age was $48.82 \pm 12.54$ years. The majority of patients $(210,89.0 \%)$ were treated with tacrolimus, and only $26(11.0 \%)$ received cyclosporine. Follow-up time ranged between 1 and 16 years (mean $7.68 \pm 4.14$ years). Mean donor age was $46.94 \pm 16.38$ years. 


\subsection{Leptin concentrations and association with clinical outcomes}

A great interindividual variability was observed in the plasma leptin concentrations of our patients. Global mean and standard deviation values were $12.42 \pm 17.80 \mathrm{ng} / \mathrm{ml}$, which are of the same order as those previously reported for kidney transplant recipients [22-24]. We could not find a significant correlation of leptin concentrations with the time elapsed since grafting $(p>0.05)$.

Figure $1 \mathrm{~A}$ shows that patients with a history of acute rejection (14.4\%) had higher values of leptin than patients without that complication did (19.92 \pm 23.72 vs $11.22 \pm 16.42 \mathrm{ng} / \mathrm{ml})$. A regression model controlling for meaningful covariates, namely donor age, hypertension in the recipient, time on dialysis, Human Leukocyte Antigen (HLA) mismatch and Homeostasis Model Assessment (HOMA) index produced a significant odds ratio (OR) with 95\% confidence intervals $(\mathrm{Cl}): 1.03(1.01-1.05), p=0.03$, indicating increased risk of rejection for patients with higher leptin plasma levels. In the same manner, patients who had experienced DGF (25.0\%) had also higher values of leptin than patients that did not have that complication (15.81 \pm 20.27 vs $11.29 \pm 16.81$; Figure $1 B$ ). However, the association was not statistically significant after controlling for other relevant covariates (hypertension in the recipient, donor age, time on dialysis and cold ischemia time) [OR=1.01 (1.00-1.03), $p=0.135]$ (Figure 1B).

Renal function, measured as estimated glomerular filtration rate (eGFR), displayed an average of $57.06 \pm 23.46 \mathrm{ml} / \mathrm{min}$ in the transplant recipients one year after grafting. Leptin concentrations negatively correlated with renal function at the time of sampling, as patients with higher leptin concentrations had much lower eGFR ( $p=0.001$; Figure 2).

\subsection{Impact of genetic variants}


Table 1 shows the distribution of the different LEPR genotypes analyzed, as well as the minor allele frequencies observed in our population. The observed frequencies did not significantly differ from those expected by the Hardy-Weinberg equilibrium (Table 1).

None of the LEPR SNPs assayed significantly affected leptin plasma levels in our series, as revealed by linear logistic regression adjusting for age at sampling, sex, body mass index (BMI), cholesterol levels and time since grafting (Supplementary Table S1).

Delayed graft function was present in $25 \%$ of patients in our series. The rs $1137101 \mathrm{G}$ allele showed an inverse association with the risk of DGF [OR $=0.50(0.27-0.91), p=0.024]$. After adjusting for other demographic and clinical covariates, the degree of the association increased significantly up to an OR of $0.42(0.22-0.81), p=0.009$ (Table 2).

With regard to the genetic association with acute rejection (experienced by $14.4 \%$ of our patients), the homozygous rs 1805094 CC genotype showed a trend towards increased risk of this complication ( $8.8 \%$ of $\mathrm{CC}$ carriers in the rejection group compared with $2.5 \%$ in the nonrejection group; $p=0.09$ ). This trend became statistically significant after controlling for other relevant variables $[\mathrm{OR}=11.38(2.15-60.16), \mathrm{p}=0.004]$ (Table 3).

Finally, a statistically significant association was observed between the rs 1137100 GG genotype and better renal function one year after grafting. GG carriers had average eGFR values of $83.41 \pm 31.36$ vs. $62.09 \pm 23.84 \mathrm{ml} / \mathrm{min}$ in AA/AG carriers. P-value for the difference was 0.010 [mean difference $=20.20(4.91-35.49)$ ] after controlling for recipient age, time in dialysis, hypertension in the recipient, HOMA index, C-reactive protein levels and leptin levels (Figure 3). 


\subsection{Haplotype study}

We next tested whether combinations of the three LEPR SNPs (rs1137100 / rs1137101 / rs18005094) could also affect clinical outcomes in renal transplantation and/or modify leptin plasma concentrations. Haplotype ${ }^{*} 1$, formed by the three wild type alleles, was the most frequent, being present in $40.5 \%$ of the population. No significant associations were observed for any of the four haplotypes when controlling for the same covariates utilized in the singleSNP study (see above).

\section{DISCUSSION}

The adipose tissue constitutes an important organ of the immune system acting through the adipocytokines. However, the role of leptin in kidney transplantation is still mostly unknown, including the relevance of the concentration variability after the transplant, its involvement in complications after grafting or the functional consequences of polymorphisms occurring in the receptor gene locus.

The first part of our study aimed to measure leptin levels in renal transplant recipients and assess their effect on clinical outcomes. We did not find significant changes in these levels related to the time elapsed since transplant. Other authors have shown that leptin concentrations decrease soon after grafting $[25-27,23]$ and increase back to pretransplant values after some years [23]. It should be noted, however, that pretransplant leptin levels were not available in our series and hence we could not measure changes vs. basal values in each individual, but rather differences between patients transplanted at different times.

Our results showed that leptin levels, measured at the time of the patient's visit to the Transplant Follow-up Unit, were directly associated with the occurrence of acute rejection. As 
we mentioned before, data on the impact of leptin on renal transplantation outcomes are very scarce. Only two research groups have previously studied this association with rejection using leptin measures at different times, although with conflicting results. Thus, Dedinska et al. [28] reported that high serum leptin concentrations before the transplant were associated with increased risk of acute rejection. In contrast, Fonseca et al. [29] could not find such association when measurements were performed in the first week after grafting. The fact that leptin levels were determined in our study with a cross-sectional design, i.e., at different times since grafting in each individual, makes our results hardly comparable with those of these two studies. In any case, an explanation for the observed higher risk of rejection could be that patients with higher leptin concentrations would presumably have an increased inflammatory response in the graft, thus increasing the risk for this complication. This same hypothesis could be applied for the occurrence of DGF. Indeed, the same study by Fonseca et al. [29], reported that higher leptin levels were related to the incidence of DGF. In our study, we could only verify a statistical trend in the same direction, but, again, the sampling took place at different times after grafting and consequently we could not confirm these previous findings based on levels measured only days after the surgery. Finally, we found that transplant recipients with higher leptin concentrations had far worse renal function (measured at the time of sampling) than other patients. It could be that the observed elevated leptin levels in these patients with poorer renal function obeyed to a reduced excretion of the protein, which is cleared from the circulation by glomerular filtration [30]. However, leptin has also been suggested to be involved in renal dysfunction, as it plays an important role in the onset and progression of glomerular endothelial proliferation, vascular damage, increased production of collagen and mesangium cells hypertrophy [31,32]. Furthermore, the Olivetti Heart prospective study just published its results arguing for a key causal role of leptin on renal risk over time [33]. We believe these two hypotheses may be 
complementary and that the observed association leptin/eGFR is likely to be a consequence of both of them.

The second part of the study focused on the impact of LEPR genetic variants. We observed that leptin concentrations did not correlate with the presence of any of the LEPR variants studied (or their combinations). Recently, it has been reported that carriers of the rs1137101 SNP showed higher leptin concentrations, although the association was exclusively observed in obese women [13]. In fact, and in line with our findings, other studies have not been able to find an effect of any of the three SNPs assayed on the circulating levels of the protein $[34,35]$, or, for that matter, of other well-studied LEPR polymorphisms [36]. In principle, a deficiency in the leptin receptor would not necessarily have to modify leptin levels. Daghestani et al. claim that this deficiency could cause leptin resistance, eventually leading to increased levels, but the authors could not find a suitable mechanism to support this hypothesis [13].

We have shown that carriers of the LEPR rs $1137101 \mathrm{G}$ allele displayed an inverse association with the risk of DGF. The acute kidney injury that leads to DGF in the first week of kidney transplantation has a strong inflammatory component [37]. It is tempting to speculate that recipients carrying the $\mathrm{G}$ variant allele would presumably have a diminished leptin receptor signaling $[38,39]$ and hence the in situ inflammatory processes related to this adipocytokine would be impaired, overall resulting in a decreased risk for the complication. To our knowledge, this is the first study to analyze these genetic associations in patients with DGF, although the SNP has been widely studied in other pathologies. For instance, our group has recently reported how this variant was associated with post-transplant diabetes mellitus, also in renal transplant recipients, and hypothesized that the leptin receptor deficiency would also be reflected in an impaired glucose-lowering function [16]. Overall, there is no consensus in 
the literature on whether the rs1137101 G variant is a "risk allele". Most likely, its effect will depend on the specific pathology and on the role leptin has in it. For instance, a recent metaanalysis reported a protective role of the G-allele in oropharyngeal cancer after examining over 35,000 subjects $[40]$.

With regard to acute rejection, carriers of the rs 1805094 CC genotype showed a significantly higher risk of acute rejection than carriers of the other genotypes did. This SNP has been far less studied than rs1137101 and its role in leptin function is unknown, so we can only speculate on what the consequences of harboring a homozygous CC genotype would be. There are studies that relate this polymorphism to the immune system [41], which could be a connection with acute rejection. In any case, we believe that the reported association with acute rejection should be interpreted with caution, as the homozygous variant genotype was carried by only eight individuals, which was translated into very wide confidence intervals for the observed odds ratio.

Finally, we found that the rs 1137100 GG homozygous genotype strongly correlated with better renal function in renal transplant recipients one year after grafting. We have shown in this work that lower leptin levels were associated with better eGFR (see above). However, as we mentioned before, no relationship could be found between the LEPR genotypes and leptin concentrations, therefore, there must be a different mechanism underlying this observation. Following the same rationale described for DGF, and assuming that a homozygous variant genotype would impair leptin receptor signaling, patients with this genotype would be less exposed to leptin-related processes in the long-term, such as endothelial proliferation in the glomerulus, vascular damage, increased collagen, cell hypertrophy, etc. [31-33], as described above. These patients would then be less prone to suffer renal damage over time resulting in improved renal function compared to that of renal transplant recipients with other genotypes. 
The work presented herein has several limitations. The sample size available was not large enough as to provide with numerous carriers of the homozygous variant genotypes, which rendered some wide, though largely significant, confidence intervals for the associations reported. In addition, we collected blood samples in a cross-sectional manner, which means that leptin levels were determined at a different time since grafting in each patient. Not having pretransplant leptin measurements precluded the confirmation of previous reports on the evolution of leptin levels after renal transplant.

In conclusion, leptin is an adipocytokine that allows the organism to adapt to the environment through inflammation and the activation of various metabolic pathways. In the present work, we have shown that leptin levels show a great variability in renal transplant recipients and that patients with higher values are more prone to complications after grafting. In addition, we observed that genetic polymorphisms in the leptin receptor gene are also able to affect clinical outcomes in these patients, suggesting that the screening of leptin-related genes may be useful to help predict post-transplant adverse events. Notwithstanding, further studies are warranted that can both include larger cohorts of renal transplant recipients and, in particular, unequivocally characterize in vitro the specific biochemical consequences of $L E P R$ polymorphisms.

\section{CONFLICT OF INTEREST}

The authors declare no conflict of interests. 


\section{ACKNOWLEDGEMENTS}

The authors want to thank Mr. Ángel Rico Barrado, technician in the Department of Physiology at the School of Medicine as well as the patients included in the study and the technical and human support provided by the Facility of Bioscience Applied Techniques of SAIUEx (financed by UEX, Junta de Extremadura, MICINN, FEDER and FSE).

Funding: This work has been supported in part by grants PI15/00804 and PI18/00745 from Instituto de Salud Carlos III, Madrid (Spain) and grants IB16014 and GR18007 from Junta de Extremadura, Mérida (Spain) and Fondo Europeo de Desarrollo Regional (FEDER) “Una manera de hacer Europa". 


\section{REFERENCES}

[1] Malyszko J, Dryl-Rydzynska T, Marcinkowski W, Prystacki T, Malyszko JS. Comorbidities on kidney transplantation waiting list relative to the status of the potential recipient. Arch Med Sci 2018; 14:941-44.

[2] Nagy K, Nagaraju SP, Rhee CM, Mathe Z, Molnar MZ. Adipocytokines in renal transplant recipients. Clin Kidney J 2016; 9:359-73.

[3] Gu P, Xu A. Interplay between adipose tissue and blood vessels in obesity and vascular dysfunction. Rev Endocr Metab Disord 2013; 14:49-58.

[4] Cumin F, Baum HP, Levens N. Leptin is cleared from the circulation primarily by the kidney. Int J Obes Relat Metab Disord 1996; 20:1120-6.

[5] Zoccali C, Mallamaci F, Tripepi G, Benedetto FA, Cutrupi S, Parlongo S, et al. Adiponectin, metabolic risk factors, and cardiovascular events among patients with end-stage renal disease. J Am Soc Nephrol 2002; 13:134-41.

[6] Briley LP, Szczech LA. Leptin and renal disease. Semin Dial 2006; 19:54-9.

[7] Komura N, Kihara S, Sonoda M, Maeda N, Tochino Y, Funahashi T, et al. Increment and impairment of adiponectin in renal failure. Cardiovasc Res 2010; 86:471-7.

[8] Chai SB, Sun F, Nie XL, Wang J. Leptin and coronary heart disease: a systematic review and meta-analysis. Atherosclerosis 2014; 233:3-10.

[9] Head GA, Lim K, Barzel B, Burke SL, Davern PJ. Central nervous system dysfunction in obesity-induced hypertension. Curr Hypertens Rep 2014; 16:466.

[10] McNeely MJ, Boyko EJ, Weigle DS, Shofer JB, Chessler SD, Leonnetti DL, et al. Association between baseline plasma leptin levels and subsequent development of diabetes in Japanese Americans. Diabetes Care 1999; 22:65-70. 
[11] Alam A, Molnar MZ, Czira ME, Rudas A, Ujszaszi A, Kalantar-Zadeh K, et al. Serum adiponectin levels and mortality after kidney transplantation. Clinical journal of the American Society of Nephrology : CJASN 2013; 8:460-7.

[12] Gervasini G, Garcia-Pino G, Mota-Zamorano S, Luna E, Garcia-Cerrada M, Tormo MA, et al. Association of polymorphisms in leptin and adiponectin genes with long-term outcomes in renal transplant recipients. Pharmacogenomics J 2019.

[13] Daghestani M, Purohit R, Daghistani M, Warsy A. Molecular dynamic (MD) studies on Gln233Arg (rs1137101) polymorphism of leptin receptor gene and associated variations in the anthropometric and metabolic profiles of Saudi women. PloS one 2019; 14:e0211381.

[14] Aijala M, Santaniemi M, Bloigu R, Kesaniemi YA, Ukkola O. Leptin receptor Arg109 homozygotes display decreased total mortality as well as lower incidence of cardiovascular disease and related death. Gene 2014; 534:88-92.

[15] Lian Y, Tang Z, Xie Y, Chen Z. Leptin receptor gene polymorphisms and risk of hypertension: a meta-analysis. Int J Clin Exp Med 2015; 8:14277-82.

[16] Mota-Zamorano S, Luna E, Garcia-Pino G, Gonzalez LM, Gervasini G. Variability in the leptin receptor gene and other risk factors for post-transplant diabetes mellitus in renal transplant recipients. Ann Med 2019:1-30.

[17] Perico N, Cattaneo D, Sayegh MH, Remuzzi G. Delayed graft function in kidney transplantation. Lancet 2004; 364:1814-27.

[18] Joosten SA, Sijpkens YW, van Kooten C, Paul LC. Chronic renal allograft rejection: pathophysiologic considerations. Kidney Int 2005; 68:1-13.

[19] Gervasini G, Garcia M, Macias RM, Benitez J, Caravaca F, Cubero JJ. CYP2C8*3 Polymorphism and Donor Age are Associated With Allograft Dysfunction in Kidney 
Transplant Recipients Treated With Calcineurin Inhibitors. J Clin Pharmacol 2013; 53:427-34.

[20] Levey AS, Greene T, Kuske J, Beck GJ, Group MS. A simplified equation to predict glomerular filtration rate from serum creatinine. J Am Soc Nephrol 2000; 11:155A.

[21] K/DOQI clinical practice guidelines for chronic kidney disease: evaluation, classification, and stratification. Am J Kidney Dis 2002; 39:S1-266.

[22] Kokot F, Adamczak M, Wiecedil, cek A, Spiechowicz U, Mesjasz J. Plasma immunoreactive leptin and neuropeptide $\mathrm{Y}$ levels in kidney transplant patients. Am J Nephrol 1999; 19:28-33.

[23] Nicoletto BB, Souza GC, Goncalves LF, Costa C, Perry IS, Manfro RC. Leptin, insulin resistance, and metabolic changes 5 years after renal transplantation. J Ren Nutr 2012; 22:440-9.

[24] Chen YC, Lee MC, Lee CJ, Hsu BG. Hyperleptinemia is associated with the aortic augmentation index in kidney transplant recipients. Ci Ji Yi Xue Za Zhi 2018; 30:152-57.

[25] Kokot F, Adamczak M, Wiecek A. Plasma leptin concentration in kidney transplant patients during the early post-transplant period. Nephrol Dial Transplant 1998; 13:2276-80.

[26] Kayacan SM, Yildiz A, Kazancioglu R, Sahin S, Sever MS, Ark E. The changes in serum leptin, body fat mass and insulin resistance after renal transplantation. Clin Transplant $2003 ; 17: 63-8$.

[27] El Haggan W, Chauveau P, Barthe N, Merville P, Potaux L, Aparicio M. Serum leptin, body fat, and nutritional markers during the six months post-kidney transplantation. Metabolism 2004; 53:614-9. 
[28] Dedinska I, Mackova N, Kantarova D, Kovacikova L, Granak K, Laca L, et al. Leptin - A new marker for development of post-transplant diabetes mellitus? J Diabetes Complications 2018; 32:863-69.

[29] Fonseca I, Oliveira JC, Santos J, Malheiro J, Martins LS, Almeida M, et al. Leptin and adiponectin during the first week after kidney transplantation: biomarkers of graft dysfunction? Metabolism 2015; 64:202-7.

[30] Barlovic DP, Zaletel J, Prezelj J. Adipocytokines are associated with renal function in patients with normal range glomerular filtration rate and type 2 diabetes. Cytokine $2009 ; 46: 142-5$.

[31] Ambarkar M, Pemmaraju SV, Gouroju S, Manohar SM, Bitla AR, Yajamanam N, et al. Adipokines and their Relation to Endothelial Dysfunction in Patients with Chronic Kidney Disease. J Clin Diagn Res 2016; 10:BC04-8.

[32] Ding N, Liu B, Song J, Bao S, Zhen J, Lv Z, et al. Leptin promotes endothelial dysfunction in chronic kidney disease through AKT/GSK3beta and beta-catenin signals. Biochem Biophys Res Commun 2016; 480:544-51.

[33] D'Elia L, Manfredi M, Perna L, lacone R, Russo O, Strazzullo P, et al. Circulating leptin levels predict the decline in renal function with age in a sample of adult men (The Olivetti Heart Study). Intern Emerg Med 2019; 14:507-13.

[34] Lopez-Quintero A, Garcia-Zapien AG, Flores-Martinez SE, Diaz-Burke Y, GonzalezSandoval CE, Lopez-Roa RI, et al. Contribution of polymorphisms in the LEP, LEPR and RETN genes on serum leptin and resistin levels in young adults from Mexico. Cell Mol Biol (Noisy-le-grand) 2017; 63:10-18.

[35] Foucan L, Bassien-Capsa V, Rambhojan C, Lacorte JM, Larifla L. Influence of K656N Polymorphism of the Leptin Receptor Gene on Obesity-Related Traits in Nondiabetic Afro-Caribbean Individuals. Metab Syndr Relat Disord 2019; 17:197-203. 
[36] Hao JQ, Zhang QK, Zhou YX, Chen LH, Wu PF. Association between circulating leptin concentration and G-2548A gene polymorphism in patients with breast cancer: a meta-analysis. Arch Med Sci 2019; 15:275-83.

[37] Mannon RB. Delayed Graft Function: The AKI of Kidney Transplantation. Nephron $2018 ; 140: 94-98$.

[38] Banks AS, Davis SM, Bates SH, Myers MG, Jr. Activation of downstream signals by the long form of the leptin receptor. J Biol Chem 2000; 275:14563-72.

[39] Stratigopoulos G, LeDuc CA, Matsuoka N, Gutman R, Rausch R, Robertson SA, et al. Functional consequences of the human leptin receptor (LEPR) Q223R transversion. Obesity (Silver Spring) 2009; 17:126-35.

[40] Rong G, Tang W, Wang Y, Qiu H, Chen S. Investigation of leptin receptor rs1137101 G>A polymorphism with cancer risk: evidence from 35936 subjects. Biosci Rep 2019; 39.

[41] Wieczorek S, Holle JU, Bremer JP, Wibisono D, Moosig F, Fricke H, et al. Contrasting association of a non-synonymous leptin receptor gene polymorphism with Wegener's granulomatosis and Churg-Strauss syndrome. Rheumatology (Oxford) 2010; 49:907-14. 


\section{FIGURE LEGENDS}

Figure 1. Distribution of leptin levels according to the occurrence of acute rejection $(A)$ or delayed graft function (B). ${ }^{*} \mathrm{p}<0.05$

Figure 2. Correlation between leptin plasma concentrations and renal function at the time of sampling.

Figure 3. Estimated glomerular filtration rate (eGFR) values one year after grafting according to the LEPR rs1137100 genotype. ${ }^{*} p<0.05$ 
TABLES 
Table 1. Genotypic and allelic frequencies in 236 renal transplant recipients.

\begin{tabular}{lccccc}
\hline \multicolumn{1}{l}{ Polymorphism /genotype } & N & $\%$ & MAF & HWE \\
\hline & AA & 135 & 57.2 & & \\
LEPR rs1137100 & AG & 90 & 38.1 & 0.237 & 0.475 \\
& GG & 11 & 4.7 & & \\
\hline & AA & 83 & 35.2 & & \\
LEPR rs1137101 & AG & 116 & 49.2 & 0.403 & 0.787 \\
& GG & 37 & 15.7 & & \\
\hline LEPR rs1805094 & GG & 155 & 65.7 & & \\
& GC & 73 & 30.9 & 0.189 & 1.000 \\
\hline & CC & 8 & 3.4 & & \\
\hline
\end{tabular}

$\mathrm{N}$, number of subjects; MAF, minor allele frequency; HWE, Hardy-Weinberg equilibrium 
Table 2. Logistic regression model for the influence of LEPR rs1137101 on the incidence of delayed graft function.

\begin{tabular}{lcccccc}
\hline & B & SE & Wald & OR & Cl & p \\
\hline LEPR rs1137101 G variant & -0.864 & 0.33 & 6.846 & 0.42 & $0.22-0.80$ & 0.009 \\
Donor age & 0.017 & 0.011 & 2.516 & 1.01 & $0.99-1.03$ & 0.113 \\
Time on dialysis & 0.091 & 0.034 & 7.034 & 1.09 & $1.02-1.17$ & 0.008 \\
Cold ischemia time & -0.007 & 0.023 & 0.111 & 0.99 & $0.95-1.03$ & 0.740 \\
Hypertension (recipient) & -0.774 & 0.358 & 4.668 & 0.46 & $0.22-0.93$ & 0.031 \\
Leptin concentrations & 0.013 & 0.009 & 2.168 & 1.01 & $0.99-1.03$ & 0.141 \\
Constant & -1 & 0.758 & 2.905 & 0.27 & - & 0.088 \\
\hline
\end{tabular}

$\mathrm{B}$, regression coefficient; $\mathrm{SE}$, standard error; OR, odds ratio; $\mathrm{Cl}, 95 \%$ confidence interval 
Table 3. Logistic regression model for the influence of LEPR rs1805094 on the incidence of acute rejection.

\begin{tabular}{lcccccc}
\hline & B & SE & Wald & OR & Cl & p \\
& & & & & & \\
\hline LEPR rs1805094 CC & 2.432 & 0.850 & 8.191 & 11.378 & $2.15-60.16$ & 0.004 \\
Donor age & 0.002 & 0.015 & 0.015 & 1.002 & $0.97-1.03$ & 0.902 \\
Time on dialysis & 0.077 & 0.041 & 3.577 & 1.080 & $0.99-1.17$ & 0.059 \\
HLA mismatch & 0.077 & 0.484 & 0.026 & 1.080 & $0.42-2.79$ & 0.873 \\
Hypertension (recipient) & 0.607 & 0.613 & 0.981 & 1.834 & $0.55-6.09$ & 0.322 \\
Leptin concentrations & 0.028 & 0.012 & 5.822 & 1.028 & $1.01-1.05$ & 0.016 \\
HOMA index & 0.067 & 0.093 & 0.507 & 1.069 & $0.89-1.28$ & 0.476 \\
Constant & -3.769 & 1.120 & 11.323 & 0.023 & - & 0.001 \\
\hline
\end{tabular}

$B$, regression coefficient; SE, standard error; OR, odds ratio; $\mathrm{Cl}, 95 \%$ confidence interval 
SUPPLEMENTARY TABLES

Supplementary Table S1. Association of leptin concentrations with the LEPR SNPS studied.

\begin{tabular}{lccccc}
\hline Polymorphism & Genotype & N & Mean & SD & p \\
\hline LEPR rs1137100 & AA & 135 & 11.86 & 17.90 & \\
& AG & 90 & 13.63 & 18.08 & 0.691 \\
& GG & 11 & 9.31 & 14.64 & \\
\hline LEPR rs1137101 & AA & 83 & 11.7 & 18.84 & \\
& AG & 116 & 11.69 & 15.62 & 0.964 \\
\hline LEPR rs1805094 & GG & 37 & 16.23 & 21.45 & \\
& GG & 155 & 12.43 & 16.72 & \\
& GC & 73 & 13.17 & 20.70 & 0.377 \\
& GC & 8 & 5.53 & 7.62 & \\
\hline
\end{tabular}

$\mathrm{N}$, number of patients; SD, standard deviation. 


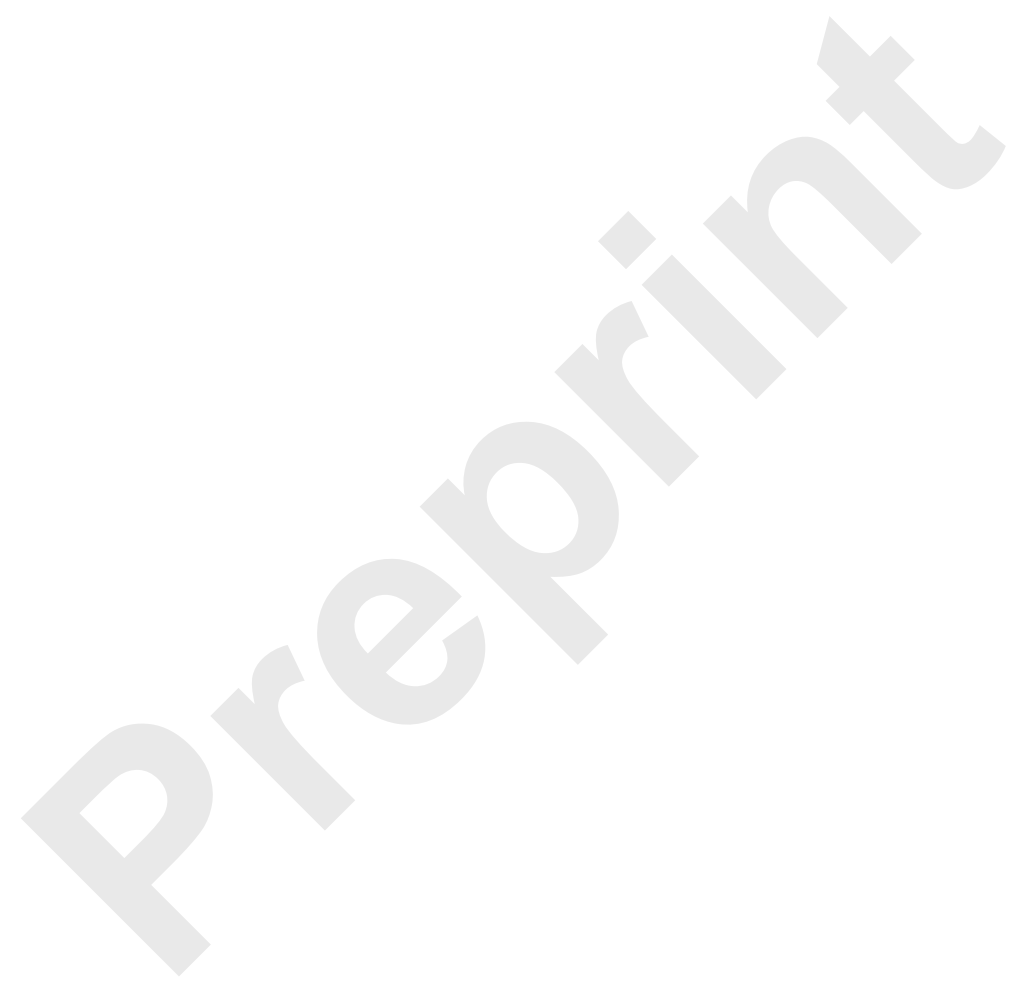


A

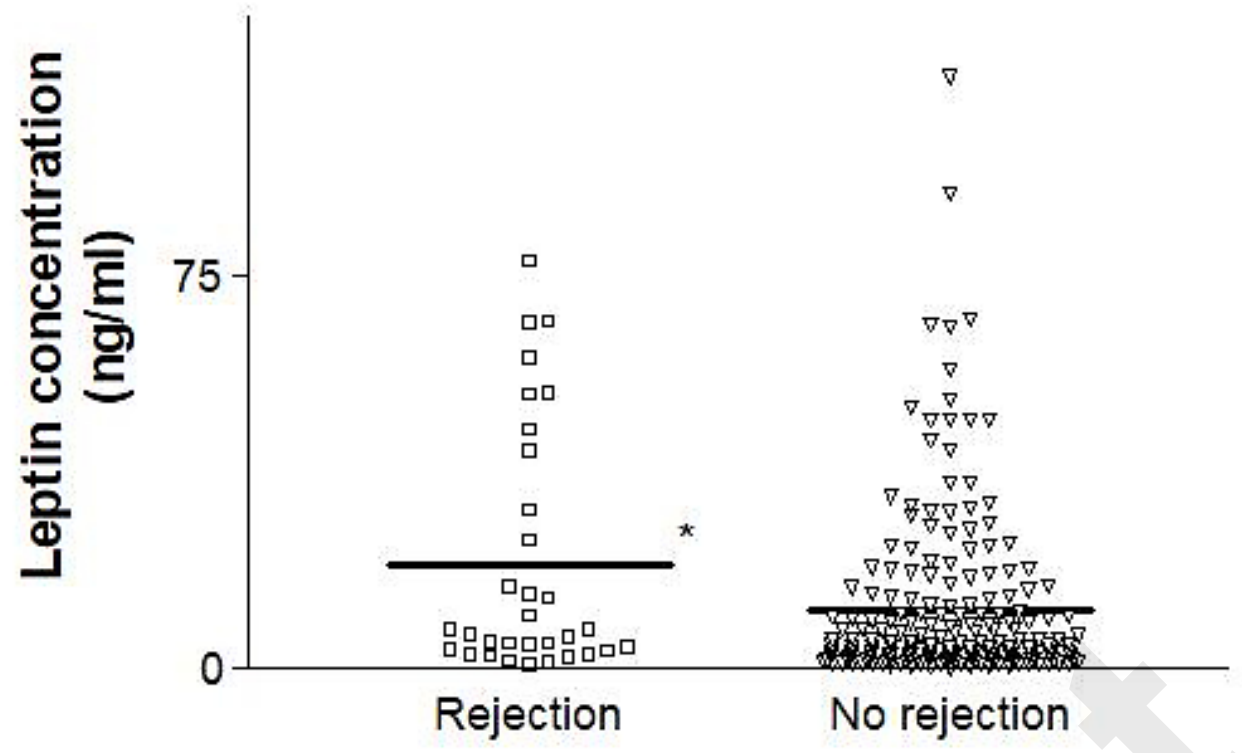

B

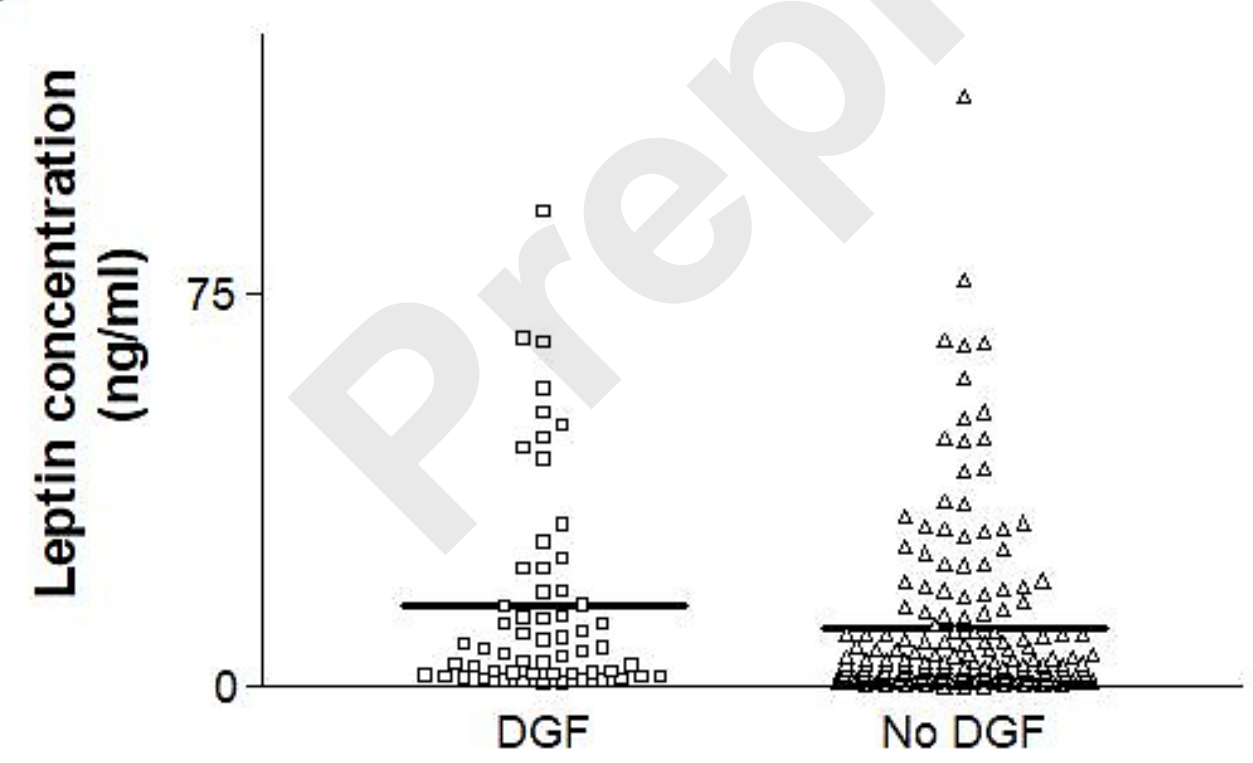

Distribution of leptin levels according to the occurrence of acute rejection (A) or delayed graft function (B). " $p<0.05$ 


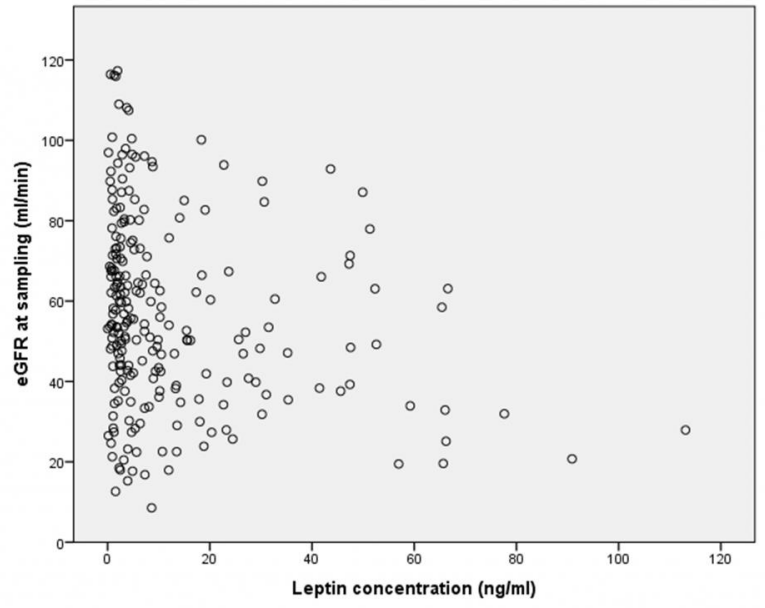

Correlation between leptin plasma concentrations and renal function at the time of sampling. 


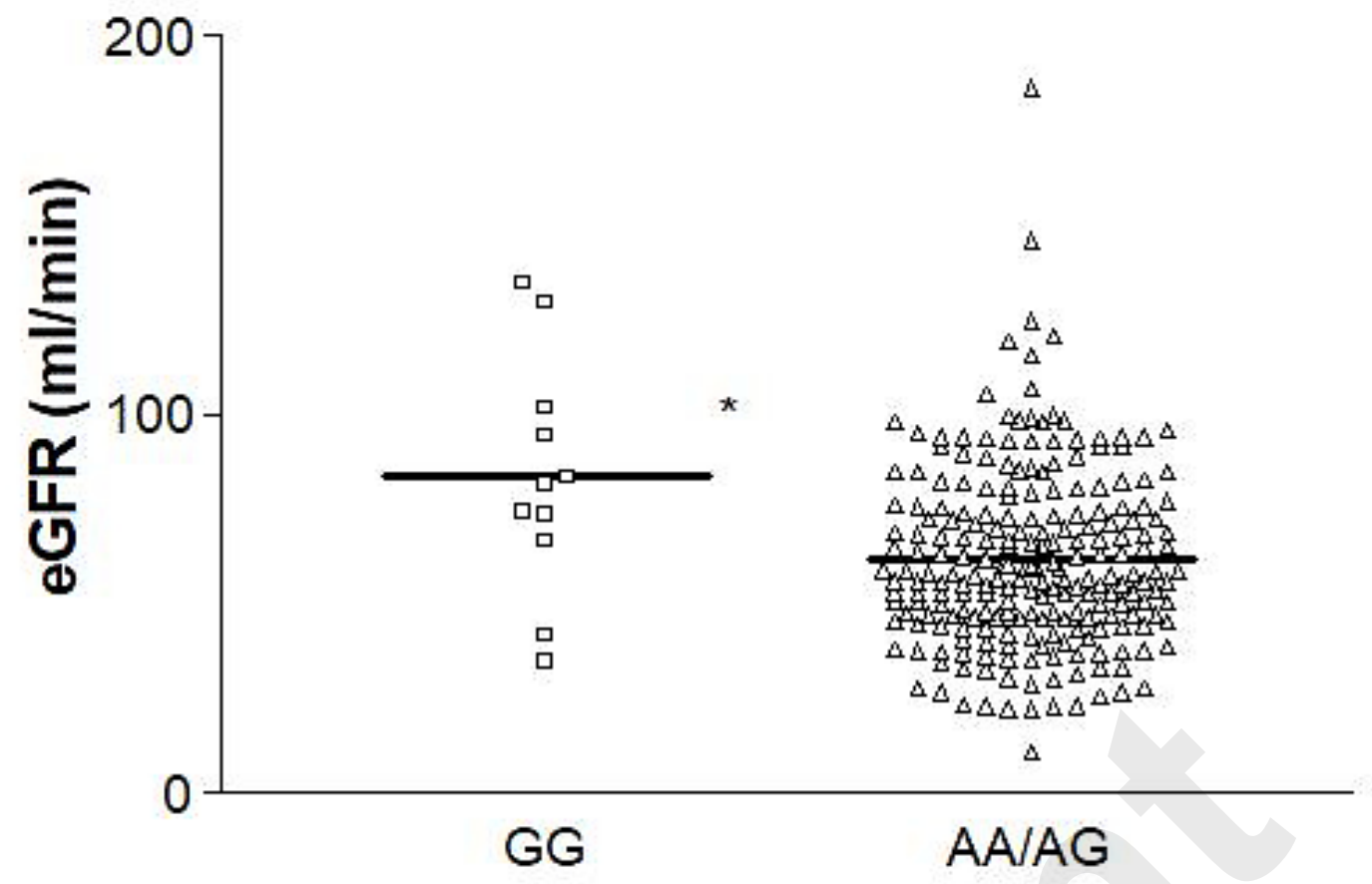

Estimated glomerular filtration rate (eGFR) values one year after grafting according to the LEPR rs1137100 genotype. * $p<0.05$ 